\title{
Clinical outcomes and Prognostic factors in children with B-Lymphoblastic Lymphoma (pB-LBL) treated on Modified BFM-90 protocol: experience from a tertiary cancer care center in India
}

Kalasekhar Vijayasekharan ${ }^{1}$, Anand $\mathrm{KC}^{1}$, Maya Prasad ${ }^{1}$, Chetan Dhamne ${ }^{1}$, Nirmalya Roy Moulik $^{1}$, Tanuja Sheth ${ }^{2}$, Epari Sridhar ${ }^{2}$, Siddhartha Laskar ${ }^{1}$, Seema Kembhavi ${ }^{1}$, Sneha Shah $^{2}$, Sumeet Gujral ${ }^{1}$, Gaurav Narula ${ }^{2}$, and Sripad Banavali ${ }^{1}$

${ }^{1}$ Tata Memorial Hospital

${ }^{2}$ Tata Memorial Centre

January 25, 2021

\begin{abstract}
Background: Pediatric B-Lymphoblastic lymphoma(pB-LBL) is a rare entity, and appropriate treatment for pB-LBL is not well defined. While intensive Acute Lymphoblastic leukemia(ALL) type regimens achieve long term event free survival of $90 \%$ across western co-operative group trials, published data from Asian studies on long term outcomes in pB-LBL are scarce. We evaluated the outcomes and prognostic factors of pediatric B-LBL patients treated at our center. Methods: We retrospectively analyzed the data of pediatric B-LBL patients treated between January 2010 and December 2017 on a uniform protocol(modified BFM 90). Patients were evaluated for early response post-induction and monitored for toxicity and long term outcomes. Kaplan-Meier method was used to estimate the event free survival(EFS) and overall survival(OS). Cox regression models were performed to identify prognostic factors. Results: Of 21 patients who received treatment on the modified BFM 90 protocol, 17(81\%) were alive in remission, $3(14 \%)$ had relapse, and $1(4 \%)$ had treatment-related mortality(TRM) while in remission. Two of 3 relapsed patients subsequently expired. With a median follow-up of 66 months(range 6-114), 5-year Event free survival(EFS) and overall survival(OS) were $80 \%$ (95\% CI:71-89\%) and 91\% (95\% CI:85-97\%), respectively. While delayed presentation ([?]3 months) had inferior EFS(p-0.030), patients with elevated baseline Lactate Dehydrogenase(LDH) had a worse OS(p-0.037). Age, gender, site of origin, stage, and post-induction response had no bearing on outcome. Conclusions: Outcomes of pB-LBL patients treated on modified BFM 90 protocol are excellent. Higher disease burden manifested by elevated baseline LDH and delayed presentation([?]3 months) portend poorer survival.
\end{abstract}

\section{Introduction:}

Lymphoblastic Lymphoma (LBL) is the second most common Non-Hodgkin lymphoma (NHL) among children, of which T-Lymphoblastic Lymphoma (T-LBL) accounts for 75-85\% of cases and B-Lymphoblastic Lymphoma accounts (B-LBL) for the remaining ${ }^{1}$. B-LBL is morphologically indistinguishable from B- acute lymphoblastic leukemia (B-ALL) with a homogenous proliferation of medium-sized cells with high nuclearcytoplasmic ratio expressing pan-early-B cell markers, TdT, HLA-DR with variable expressivity for CD10 and CD34 ${ }^{2}$. Pediatric B-LBL(pB-LBL) usually presents at a younger age $(<6$ years $)$ with lower stage disease and rare mediastinal and marrow involvement compared to T-LBL ${ }^{3}$. With the efforts of major international co-operative groups, current overall outcomes of pB-LBL exceed $80 \%^{4}$. However, salvage rates among relapsed/refractory pB-LBL patients remain poor ${ }^{5}$. Further improvement in outcomes of pB-LBL is marred by the overall low incidence of LBL and the lack of substantial tissue specimens for biological studies ${ }^{4}$. 
Robust prognostic markers such as cytogenetic translocations and minimal residual disease (MRD) detection which aid in treatment optimization in children with B-acute lymphoblastic leukemia (B-ALL) are still lacking for pB-LBL. Also, morphologic response criteria assessed by X-rays/CT-Scans are not uniform across various LBL trials, especially for pB-LBL patients. The role of minimal disseminated disease (MDD) detection and functional imaging such as FDG-PET/CT in the management of pB-LBL is still evolving ${ }^{6,7}$. Ongoing trials in LBL focus on identifying specific prognostic factors necessitating escalation of treatment or addition of novel therapies among high-risk subsets to improve outcome, as well as de-escalation among low-risk patients to avoid exposure to untoward toxicity. Here we report the clinical profile and long-term outcomes of pB-LBL patients treated at our center using a uniform strategy and identified factors that affect their outcomes.

\section{Materials and Methods:}

\section{Patients:}

This was a retrospective analysis of consecutively treated children aged [?]15 years with newly diagnosed B-LBL at Tata Memorial Hospital, Mumbai, India between January 2010 and December 2017. The last follow-up data of patients were censored on $1^{\text {st }}$ July 2020 . This retrospective audit was approved by the institute ethics committee.

\section{Diagnosis and Staging:}

The diagnosis of B-LBL was confirmed by expert haematopathologists on biopsy specimens based on the standard morphologic and immunophenotypic criteria. Staging workup included Whole-body PET/CT or Contrast-enhanced computerized tomography (CT) scans of thorax, abdomen, and pelvis, bilateral bone marrow aspiration/biopsy and cerebrospinal fluid analysis. Baseline marrow flowcytometric minimal disseminated disease (MDD) assessment by 8 to 10 color acute leukemia panel (>5lakh events) was done and analyzed using Kaluza version 1.3. Modified St. Jude classification was used for staging.

\section{Treatment :}

All patients were treated uniformly on a modified BFM-90 protocol ${ }^{8}$. Figure- 1 depicts the treatment schema used in the study. Following a 7-day prednisolone pre-phase, phase I 4-drug "induction" was delivered over 4weeks, followed by phase II "induction" over 4-weeks; phase M contained 4-cycles of high-dose methotrexate administered as a $24 \mathrm{hrs}$ continuous infusion; Re-intensification phase containing a truncated re-induction and consolidation over 6 -weeks, was administered to all pB-LBL patients with advanced-stage disease (Stage III and IV). Also, a decision to give re-intensification phase in limited-stage disease (Stage-I/II) with baseline detectable MDD and/or PET positivity in the marrow and to administer local irradiation for persisting residual disease post-induction phase II irrespective of the stage was taken in Multidisciplinary tumor boards discussions on a case-by-case basis. Both limited and advanced-stage B-LBL patients were given oral maintenance chemotherapy for a period of 2years. Central Nervous System chemoprophylaxis included high dose methotrexate and intrathecal methotrexate. Until 2013, prophylactic cranial irradiation (12.6 Gy) was administered for CNS-1 \& 2 status, and this was discontinued thereafter, while CNS-3 patients, continued to receive therapeutic cranial irradiation (18 Gy) after re-intensification phase. Patients with testicular involvement at presentation and having residual disease persisting post-induction phase II (D64) received testicular irradiation (24 Gy) .

\section{Response assessment:}

An interim response assessment was done post completion of induction phase I (D35) either with PET-CT or by using conventional imaging techniques. Care was taken to also include the same imaging modality that was used in initial assessment for ease of comparison. Complete response (CR) was defined as the absence of all clinical and cytological evidence of disease with $>70 \%$ reduction in diameter of all the lesions assessed by either X-ray/CT/MRI or a complete metabolic response with Deauville's score of 1-3 in PET-CT. Partial response (PR) was defined as Clinico-cytologic response with $<70 \%$ decrease in any of the lesion with no new lesions by X-ray/CT/MRI or a PET positivity with Deauville's score 4 with a decrease in metabolic activity 
compared to baseline scans and Progressive disease (PD) as clinico-radiologic worsening with Duvellie's score 5 and/or appearance of new leisions in interim PET. Patients who had PR in the post-induction phase I (D35), response assessment were re-assessed at the end of the induction phase II (D64). Patients who had baseline bone marrow involvement with $>5 \%$ blasts underwent reassessment only by flow-cytometric minimal residual disease (MRD) post-induction phase I (D35). Post-phase I induction MRD was considered positive when the levels $>0.01 \%$. In CNS-3 patients, CSF analysis was repeated weekly along with Intra-thecal methotrexate administration until two consecutive CSF analysis remained negative for blasts.

\section{Statistical Analysis:}

Clinical and demographic profiles were analyzed using descriptive statistics. A comparison of proportions was done with $\mathrm{chi}^{2}$ or Fisher's exact tests. Event free survival (EFS) was calculated from date of diagnosis to the occurrence of any event (relapse, progression, death, second malignancy) or till last follow-up date. Overall survival was calculated from the date of diagnosis to date of death due to any cause or till last follow-up date. Kaplan-Meier's method was used to calculate the survival estimates. Cox regression model was used to compare the survival curves. All statistical tests were two-sided and a p-value of less than 0.05 was considered significant. Statistical analysis was performed using SPSS Statistics for Windows, version 24 (SPSS IncVR,Chicago, IL).

\section{Results:}

\section{Demographic and Clinical profile (Table 1):}

During the study period, 227 children were registered with a diagnosis of Lymphoblastic Lymphoma (LBL), of which 25 (11\%) children had B-Lymphoblastic lymphoma (B-LBL). Only $21 \mathrm{pB}-\mathrm{LBL}$ patients (2-refused treatment, 2-came for only a second opinion) started treatment at our center with a male to female ratio2.5:1. The median age of the study cohort was 6-years (range:1-14 years). The median symptom duration at presentation was 2 months (range: 1-8 months). Subcutaneous soft tissue mass 11 (52\%) and Osteolytic bone lesions $4(19 \%)$ were the most common presentation. Other rare presentations included visceral disease involvement in $14 \%(3 / 21 ; 2$-mesticular mass, 1-Right atrial mass), isolated lymph nodal enlargement in 1(5\%),mediastinal mass in 1(5\%) and Subdural mass in 1(5\%). Advanced disease stage was noticed in $62 \%$ (13/21; Stage III- 9, Stage IV-3 ) and rest 38\% (9/21; Stage I-6, Stage II-3) had limited stage disease. Among 3 patients with stage IV disease, only 1 had morphologic marrow involvement $>5 \%$ blasts without CNS disease ( $12 \%$ bone-marrow blasts morphologically with flow-cytometry based Minimal Detectable Disease (MDD)-2.7\%), and the other 2 had only CNS involvement (1-Subdural intracranial mass with no blasts in CSF, 1-Leptomeningeal involvement with CSF positive for blasts). In the study cohort, $8(38 \%)$ had non-morphologic marrow involvement ( $<5 \%$ blasts) with flow-cytometric MDD in 4 (2 out of 4 having additional PET positive marrow) and isolated PET positive marrow without MDD in the remaining 4. Median flowcytometric MDD was $0.4 \%$ (range: $0.0009 \%$ to $2.7 \%$ ). Of the 4 -patients with marrow MDD, 2 patients had Stage I disease (PET negative marrow) and the other 2 had advanced-stage disease (1-Stage III, 1-Stage IV) with PET positive marrow. All 4 patients with isolated PET positive marrow without MDD had advanced-stage disease (Stage III). Baseline lactate dehydrogenase (LDH) was elevated (>1 ULN) in 8 patients with a median of $285.5 \mathrm{U} / \mathrm{L}$ (Reference lab range:110-290 U/L).

\section{Treatment, response, and toxicity:}

All patients in the study received modified BFM-90 chemotherapy per-protocol. In the limited stage disease, all except two stage-1 patients (patient-4 and patient-13) received the re-intensification phase. Almost all patients (20/21) had baseline staging assessment by PET-CT, of which, 14 patients (3 Stage-I, 3 Stage-II, 7 Stage III, 1 Stage IV) had a post-phase I induction response assessment by PET/CT. Rest 7/21 (3 Stage-I, 2 Stage III, 2 Stage IV) underwent interim assessment by non-functional imaging modalities. At the end of phase I induction (D35), 15/21(71\%) patients achieved a CR, and 6/21(29\%) had PR. After completion of induction phase II (Day 64), 19/21(90\%) patients achieved CR, and 2/21 (10\%) had residual disease. A stageIV, CNS-3 patient (patient-8) with residual testicular disease post-phase II induction was given testicular irradiation (24Gy) besides cranial irradiation (18Gy). Two stage-III patients (patient-1 and patient-20) with 
bony primary who had only partial response post-induction phase I and II received irradiation to their residual bony lesion (55.6Gy and 45Gy, respectively). The toxicities resulting in hospital admission were noted during the study period. Eight patients had a total of $15 \mathrm{FN}$ episodes. Other toxicities included recurrent urinary tract infection in a child with paraspinal mass and bladder dysfunction, uncomplicated dengue fever, probable fungal pneumonia. One patient had a transient ischemic attack (TIA) as a complication of high dose methotrexate infusion (Patient-6). In the whole cohort, there was one toxic death in remission secondary to culture-negative severe septicemia during the re-intensification phase (Patient-6).

\section{Outcomes and Prognostic Factors (Figure 1, Table 2)}

The median follow-up of the entire study cohort was 66months (range:6-114 months). The 5-year EFS and OS of the cohort were $80 \%$ (95\%CI:71-89\%) and 91\% (95\%CI: 85-97\%), respectively (Figure 2\&3). The 5 -year EFS/OS in limited stage disease were 78\% (95\%CI: 65-91\%)/89\% (95\%CI:79-99\%) and in advanced stage $80 \%$ (95\%CI: 68-92\%) /91\% (95\%CI: 83-98\%) respectively. Among the study cohort of 21 patients, there were three relapses and one toxic death. Three patients relapsed at a median of 37months (range:19-55 months) from diagnosis, of which two expired secondary to progression of the disease and one patient is alive in remission following salvage chemotherapy. None of the relapsed patients in the study cohort underwent hematopoietic stem cell transplant due to socio-economic constraints. Among the three relapsed patients only one had partial response (PR) post-induction.

Patients who presented late ([?]3 months) from symptom onset had a significantly poorer 5 -year event free survival of $44.4 \pm 22.2 \%$ compared to patients presenting early (<3months) $93.3 \pm 6.4 \%$ (p-0.030). Also, there was a trend towards poorer overall survival among pB-LBL patients presenting with osteolytic primaries compared to soft tissue lesions (5-year OS-75 $\pm 21.7 \%$ vs $90.9 \pm 8.7 \%$; p- 0.080 ). None of the patients with MDD detected in the marrow baseline relapsed. Elevated serum lactate dehydrogenase (LDH) $>1$ ULN at diagnosis was associated with significantly poorer overall survival (p-0.032). Age, gender, stage, baseline bone marrow involvement, and Post-induction response had no bearing on survival outcomes.

\section{Discussion:}

This is the first report on the outcomes of a large prospective cohort of pediatric B-LBL from a refererral center in India. Pediatric B-LBL patients in the study received a modified BFM 90 protocol. While all children with advanced stage disease received re-intensification, administration of re-intensification for limited-stage disease and local radiotherapy (LRT) to primary was decided by the multidisciplinary team on a case-to-case basis. From 2014, Cranial Radiotherapy (CRT) of 18Gy was given only to CNS-3 patients. For the 21 pB-LBL patients, 5-year EFS and OS rates of $80 \%$ and $91 \%$ using the modified BFM 90 protocol were in accordance with major reported series in pB-LBL so far $^{3,9-13}$. Age, gender, stage, baseline bone marrow involvement (either morphologic or by MDD/PET), and the post-phase I induction response do not seem to influence survival rates in our pB-LBL patients. This study reiterates the fact that intensive intrathecal injections in addition to high dose methotrexate (HDMTX) can safely replace prophylactic cranial irradiation (CRT) as described in major studies on childhood $\mathrm{LBL}^{8,14,15}$. Identifying prognostic factors is important due to poor prognosis at relapse, but is a challenge in childhood B-lymphoblastic lymphoma due to the rarity of the disease ${ }^{4}$.

Delayed presentation as a cause of poor outcomes in childhood lymphoblastic lymphoma has been reported earlier ${ }^{16}$, and attributed to the diagnostic delay in developing countries. Two-third of the pB-LBL patients in our cohort with delayed presentation (4/6) had advanced-stage disease. Although there is a paucity of data on diagnostic delays in childhood lymphoblastic lymphoma and its impact on outcomes, we presume this poor outcome may be due to the development of biologic resistance with the accumulation of clonal genetic aberrations over time ${ }^{17,18}$. Elevated baseline LDH (>1ULN), an indicator of tumor bulk was also found to be significantly associated with poorer overall survival in our pB-LBL cohort (p-0.032). In high-grade NHL like Burkitt's lymphoma and DLBCL, elevated baseline LDH (>2ULN) is a well-known predictor of overall poor outcome. Major studies on childhood lymphoblastic lymphoma were predominantly involving T-LBL and they found no direct association of baseline LDH with survival ${ }^{9,10}$. Also, childhood B-LBL is very rare 
which presents usually with localized stages with normal baseline LDH. Elevated baseline LDH in pB-LBL may be a potential biomarker of poor survival.

There was a trend towards poorer overall survival among advanced stage B-LBL patients presenting with osteolytic primaries compared to soft tissue lesions ( $\mathrm{p}-0.08)$. Major pediatric co-operative groups noticed excellent outcomes of localized lymphoblastic lymphoma arising from bones ${ }^{10,19,20}$. However, 3/4 B-LBL patients with osteolytic primary had advanced-stage disease in our study, and 2 among them relapsed (1medullary and 1-extramedullary relapse). One patient with localized iliac bone primary (patient-13) and another with Stage III disease and a tibial primary (patient-20) are alive in remission. Radiotherapy played a significant role in treatment regimens of primary bone lymphomas in the past ${ }^{21,22}$, but was associated with an increased risk of secondary malignancies such as sarcomas in the radiation field. As various studies have shown favorable survival rates without radiation, this modality is no longer included in standard treatment plans of primary bony lymphomas ${ }^{10}$. In our study, patient- 1 and patient-20 with bony primaries received local radiotherapy in-view of persistent residual disease post- induction phase II.

Mussolin et $\mathrm{al}^{6}$ found flow cytometry detectable MDD in $23 \%$ of pB-LBL patients without cytological BM involvement. They also noticed a higher level of MDD $(>3 \%)$ to be associated with a poorer event free survival in lymphoblastic lymphoma in children. Twenty percent (4/20) of our pB-LBL patients had baseline flow cytometry detectable MDD without cytological BM involvement (median-0.4\%; Range:0.0009-0.7\%). One Stage-IV B-LBL patient (patient-12) with $12 \%$ morphologic marrow involvement had $2.7 \%$ MDD by flow cytometry. We did not notice a difference in outcome in our B-LBL patients with or without baseline BM MDD which may be explained by the lower log of MDD noticed in our cohort compared to Mussolin et al. and also due to the potential use of re-intensification phase in limited-stage patients with baseline BM MDD.

Three relapses $(14 \%)$ and one toxic death (5\%) due to severe gram-negative septicemia remained the reason for treatment failure in our study. One patient with late testicular relapse is alive in remission post-salvage therapy and the rest $2 / 3$ relapsed patients (1-early extramedullary relapse and 1-Late isolated medullary relapse) progressed on salvage chemotherapy and died. The EORTC reported a $15 \%$ relapse/progression rate in 8/53 B-LBL patients diagnosed between 1989 and 2008. All eight patients died, seven after allogeneic HSCT, five patients due to disease progression, and three fromTRM ${ }^{3}$. Irrespective of TRM, the best chance of cure after pB-LBL relapse is with high dose chemotherapy followed by allogeneic stem cell transplant ${ }^{5,23}$. Prospective future trials utilizing Blinatumomab or CAR-T cells in addition to chemotherapy backbone may potentially reduce failures in pB-LBL patients

Although with the limitation of retrospective single center analysis on a smaller number of pediatric B-LBL patients and lack of detailed toxicity data, our study identified a few potential clinical biomarkers which may aid in the risk stratification of pB-LBL patients in future trials.

Conclusions: Pediatric B-Lymphoblastic lymphoma is a rare entity. The modified BFM 90 protocol achieves excellent outcomes in pediatric B-LBL patients treated in the LMIC setting. pB-LBL patients presenting late ([?]3months) from the first symptom onset and with elevated baseline Lactate dehydrogenase (LDH) fared poorly on this protocol.

\section{Conflict of interest:}

The authors declare that there is no conflict of interest.

\section{References:}

1. Burkhardt B, Hermiston ML. Lymphoblastic lymphoma in children and adolescents: review of current challenges and future opportunities.Br J Haematol . 2019;185(6):1158-1170. doi:10.1111/bjh.15793

2. Soslow RA, Baergen RN, Warnke RA. B-lineage lymphoblastic lymphoma is a clinicopathologic entity distinct from other histologically similar aggressive lymphomas with blastic morphology. Cancer . 1999;85(12):2648-2654. 
3. Ducassou S, Ferlay C, Bergeron C, et al. Clinical presentation, evolution, and prognosis of precursor B-cell lymphoblastic lymphoma in trials LMT96, EORTC 58881, and EORTC 58951. Br J Haematol . 2011;152(4):441-451. doi:10.1111/j.1365-2141.2010.08541.x

4. Minard-Colin V, Brugieres L, Reiter A, et al. Non-Hodgkin Lymphoma in Children and Adolescents: Progress Through Effective Collaboration, Current Knowledge, and Challenges Ahead. J ClinOncol Off $J$ Am SocClinOncol . 2015;33(27):2963-2974. doi:10.1200/JCO.2014.59.5827

5. Schmidt E, Burkhardt B. Lymphoblastic lymphoma in childhood and adolescence. PediatrHematolOncol . 2013;30(6):484-508. doi:10.3109/08880018.2013.789574

6. Mussolin L, Buldini B, Lovisa F, et al. Detection and role of minimal disseminated disease in children with lymphoblastic lymphoma: The AIEOP experience. Pediatr Blood Cancer . 2015;62(11):1906-1913. doi:10.1002/pbc. 25607

7. Nakatani K, Nakamoto Y, Watanabe K, Saga T, Higashi T, Togashi K. Roles and limitations of FDG PET in pediatric non-Hodgkin lymphoma.ClinNucl Med . 2012;37(7):656-662. doi:10.1097/RLU.0b013e318238f72b

8. Reiter A, Schrappe M, Ludwig WD, et al. Intensive ALL-type therapy without local radiotherapy provides a $90 \%$ event-free survival for children with T-cell lymphoblastic lymphoma: a BFM group report.Blood . 2000;95(2):416-421.

9. Landmann E, Burkhardt B, Zimmermann M, et al. Results and conclusions of the European Intergroup EURO-LB02 trial in children and adolescents with lymphoblastic lymphoma. Haematologica . 2017;102(12):2086-2096. doi:10.3324/haematol.2015.139162

10. Termuhlen AM, Smith LM, Perkins SL, et al. Outcome of newly diagnosed children and adolescents with localized lymphoblastic lymphoma treated on Children's Oncology Group trial A5971: a report from the Children's Oncology Group. Pediatr Blood Cancer . 2012;59(7):1229-1233. doi:10.1002/pbc.24149

11. Burkhardt B, Zimmermann M, Oschlies I, et al. The impact of age and gender on biology, clinical features and treatment outcome of non-Hodgkin lymphoma in childhood and adolescence. $\mathrm{Br} J$ Haematol . 2005;131(1):39-49. doi:10.1111/j.1365-2141.2005.05735.x

12. Termuhlen AM, Smith LM, Perkins SL, et al. Disseminated lymphoblastic lymphoma in children and adolescents: results of the COG A5971 trial: a report from the Children's Oncology Group. $\mathrm{Br} J$ Haematol . 2013;162(6):792-801. doi:10.1111/bjh.12460

13. Sandlund JT, Pui C-H, Zhou Y, et al. Effective treatment of advanced-stage childhood lymphoblastic lymphoma without prophylactic cranial irradiation: results of St Jude NHL13 study. Leukemia . 2009;23(6):1127-1130. doi:10.1038/leu.2008.400

14. Uyttebroeck A, Suciu S, Laureys G, et al. Treatment of childhood T-cell lymphoblastic lymphoma according to the strategy for acute lymphoblastic leukaemia, without radiotherapy: long term results of the EORTC CLG 58881 trial. Eur J Cancer OxfEngl 1990 . 2008;44(6):840-846. doi:10.1016/j.ejca.2008.02.011

15. Asselin BL, Devidas M, Wang C, et al. Effectiveness of high-dose methotrexate in T-cell lymphoblastic leukemia and advanced-stage lymphoblastic lymphoma: a randomized study by the Children's Oncology Group (POG 9404). Blood . 2011;118(4):874-883. doi:10.1182/blood-2010-06-292615

16. Gao Y-J, Pan C, Tang J-Y, et al. Clinical outcome of childhood lymphoblastic lymphoma in Shanghai China 2001-2010. Pediatr Blood Cancer . 2014;61(4):659-663. doi:10.1002/pbc.24848

17. Nowell PC. The clonal evolution of tumor cell populations.Science . 1976;194(4260):23-28. doi:10.1126/science.959840

18. Rushton CK, Arthur SE, Alcaide M, et al. Genetic and evolutionary patterns of treatment resistance in relapsed B-cell lymphoma.Blood Adv . 2020;4(13):2886-2898. doi:10.1182/bloodadvances.2020001696 
19. Suryanarayan K, Shuster JJ, Donaldson SS, Hutchison RE, Murphy SB, Link MP. Treatment of Localized Primary Non-Hodgkin's Lymphoma of Bone in Children: A Pediatric Oncology Group Study. J ClinOncol . 1999;17(2):456-456. doi:10.1200/JCO.1999.17.2.456

20. Lones MA, Perkins SL, Sposto R, et al. Non-Hodgkin's lymphoma arising in bone in children and adolescents is associated with an excellent outcome: a Children's Cancer Group report. J ClinOncol Off $J$ Am SocClinOncol . 2002;20(9):2293-2301. doi:10.1200/JCO.2002.06.017

21. Loeffler JS, Tarbell NJ, Kozakewich H, Cassady JR, Weinstein HJ. Primary lymphoma of bone in children: analysis of treatment results with adriamycin, prednisone, Oncovin (APO), and local radiation therapy.J ClinOncol Off J Am SocClinOncol . 1986;4(4):496-501. doi:10.1200/JCO.1986.4.4.496

22. Susnerwala SS, Dinshaw KA, Pande SC, et al. Primary lymphoma of bone: experience of 39 cases at the Tata Memorial Hospital, India.J SurgOncol . 1990;44(4):229-233. doi:10.1002/jso.2930440408

23. Gross TG, Hale GA, He W, et al. Hematopoietic stem cell transplantation for refractory or recurrent non-Hodgkin lymphoma in children and adolescents. Biol Blood Marrow Transplant J Am Soc Blood Marrow Transplant . 2010;16(2):223-230. doi:10.1016/j.bbmt.2009.09.021

\section{LEGENDS}

TABLE 1. Clinical profile and Outcomes of pediatric B-Lymphoblastic Lymphoma

TABLE 2. Univariate analysis of prognostic variables affecting survival in pB-LBL

Figure 1. Modified BFM 90 Treatment Schema used in Study (*IT- Intrathecal, \#CR-Complete response)

Figure 2. 5-year Event free survival is $80 \%$

Figure 3. 5-year Overall survival is $91 \%$ 


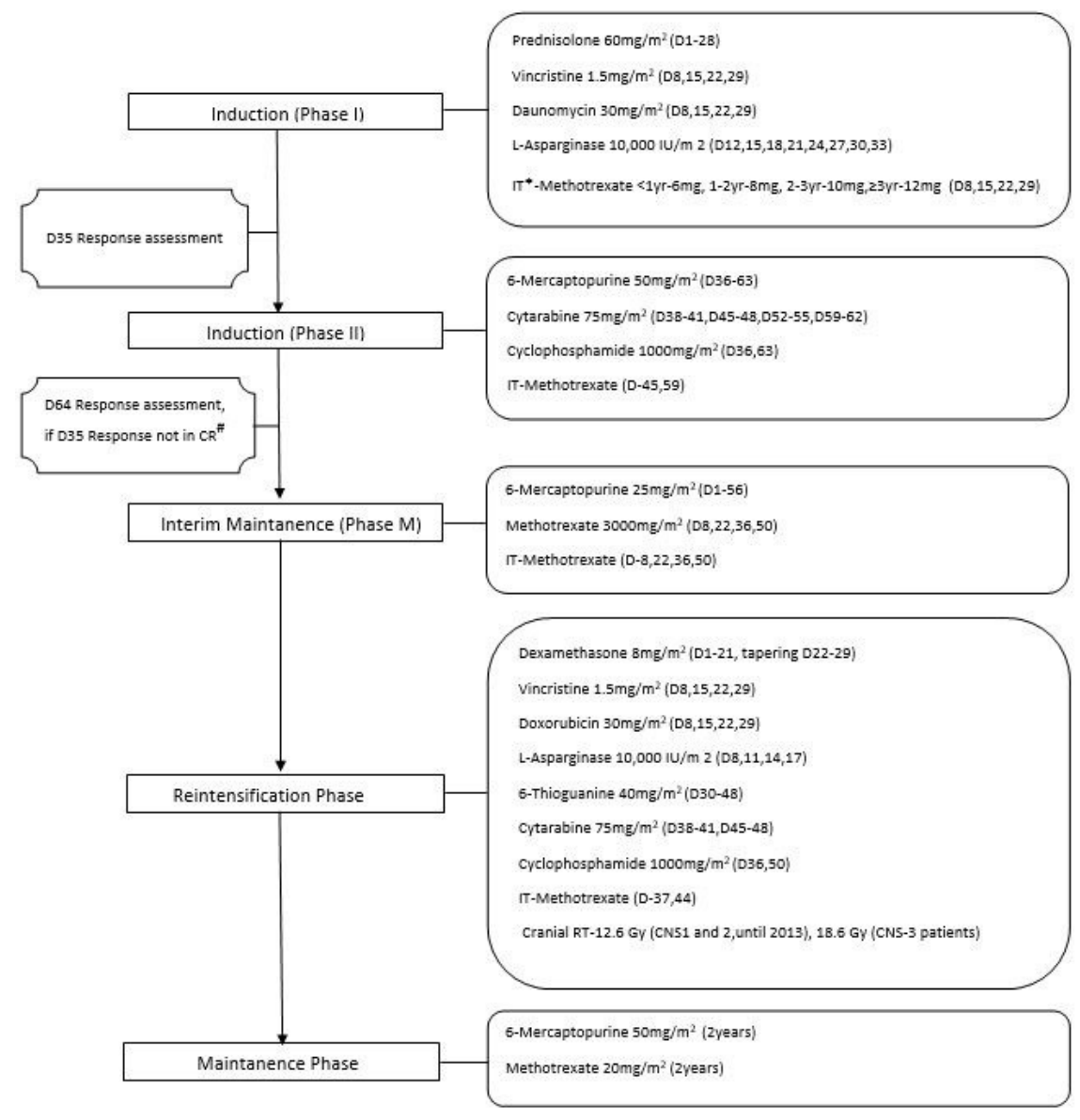



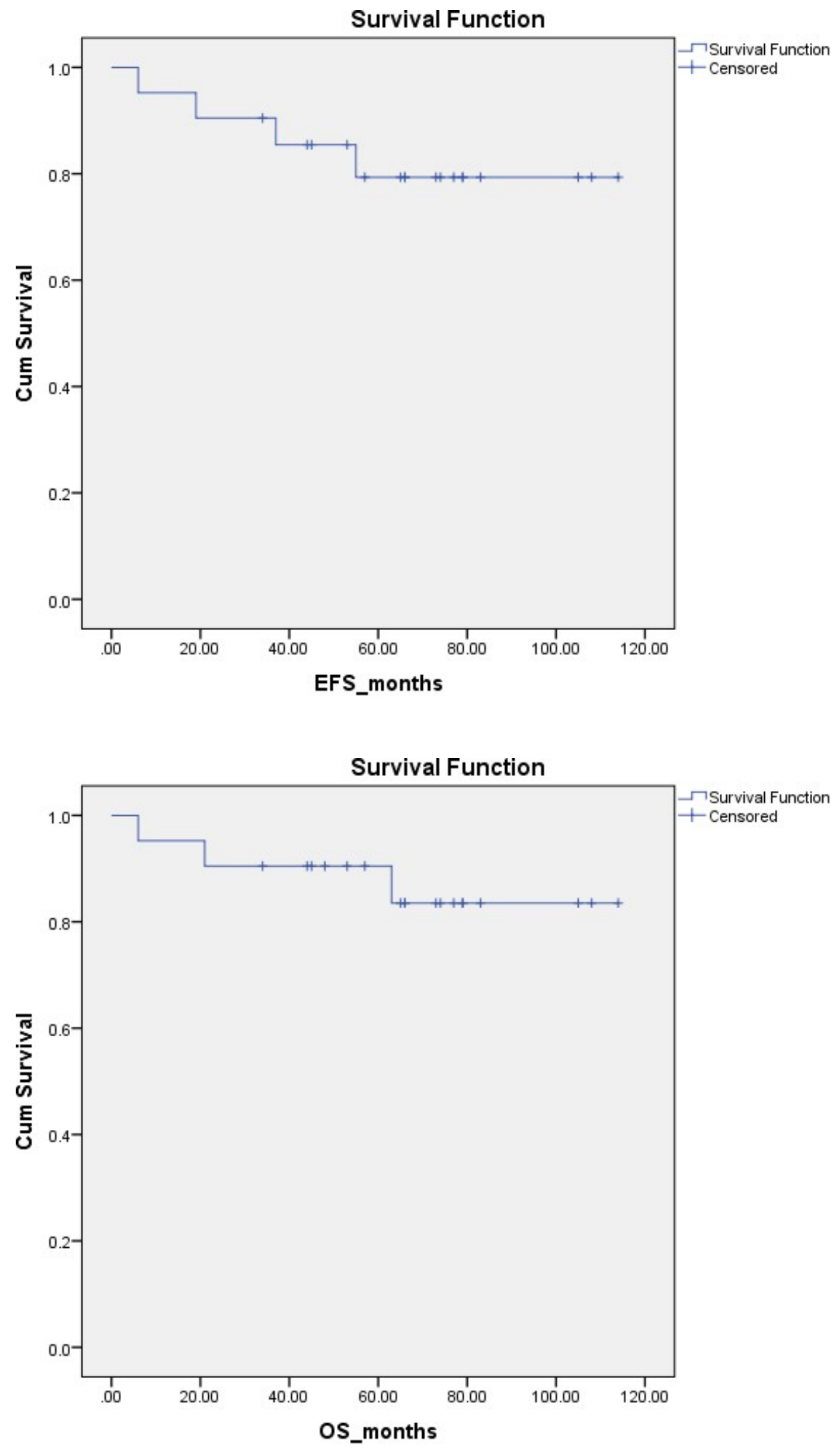

Hosted file 
Table-1.pdf available at https://authorea.com/users/329133/articles/505712-clinical-outcomesand-prognostic-factors-in-children-with-b-lymphoblastic-lymphoma-pb-lbl-treated-onmodified-bfm-90-protocol-experience-from-a-tertiary-cancer-care-center-in-india

\section{Hosted file}

Table-2.pdf available at https://authorea.com/users/329133/articles/505712-clinical-outcomesand-prognostic-factors-in-children-with-b-lymphoblastic-lymphoma-pb-lbl-treated-onmodified-bfm-90-protocol-experience-from-a-tertiary-cancer-care-center-in-india 